\title{
A desigualdade espacial do Baixo Peso ao Nascer no Brasil
}

\author{
The spatial inequality of low birth weight in Brazil
}

\author{
Marina Clarissa Barros de Melo Lima ${ }^{1}$ \\ Genyklea Silva de Oliveira ${ }^{1}$ \\ Clélia de Oliveira Lyra ${ }^{1}$ \\ Angelo Giuseppe Roncalli ${ }^{1}$ \\ Maria Angela Fernandes Ferreira ${ }^{1}$
}

\footnotetext{
${ }^{1}$ Programa de PósGraduação em Saúde Pública, Universidade Federal do Rio Grande do Norte. Av. Senador Salgado Filho 1787, Lagoa Nova. 59.056-000 Natal RN. marinaclarissa@hotmail.com
}

\begin{abstract}
Low birth weight ( $L B W$ ) is a risk factor for neonatal and infant morbidity and mortality. In Brazil the highest percentages of low birth weight occur in regions of higher socioeconomic status. The scope of this article is to ascertain the spatial distribution of low birth weight rates and the correlation with social and service indicators. The scale is ecological taking all the Brazilian states as units of analysis. The spatial analysis technique is the methodology used together with data from SINASC, IPEA and IBGE for 2009. Higher rates of low birth weight are found in the south/southeastern states (Global Moran: 0.267, $p=0.02$ ). Clusters of the highhigh type in the Southeast and of the low-low variety in states in the Amazon region are detected. The spatial inequality of low birth weight reflects the socio-economic conditions of the states. More developed regions have higher rates of low birth weight, therefore, the presence of the service and its use decrease infant mortality and increase $L B W$.
\end{abstract}

Key words Low infant birth weight, Medical geography, Social indicators
Resumo O baixo peso ao nascer (BPN) é fator de risco para a morbidade, mortalidade neonatal e infantil. No Brasil os maiores percentuais de baixo peso ao nascer ocorrem em regiões de melhor situação socioeconômica. O objetivo deste artigo é conhecer a distribuição espacial de taxas de baixo peso ao nascer e a correlação com indicadores sociais e de serviço. Desenho: ecológico, tendo os estados brasileiros como unidades de análise. Utilizou-se técnica de análise espacial, dados de 2009 do SINASC, IPEA e IBGE. Taxas maiores de baixo peso ao nascer estão nos estados da região sul/ sudeste, Moran Global: 0,267, $p=0,02$. Aglomerados do tipo alto-alto na região Sudeste e baixobaixo em estados da região da Amazônia Legal. A desigualdade espacial do baixo peso ao nascer reflete as condições socioeconômicas dos estados. Regiões mais desenvolvidas detêm maiores taxas de baixo peso ao nascer, portanto, a presença do serviço e sua utilização fazem diminuir a mortalidade infantil e aumentar o BPN.

Palavras-chave Recém-nascido de Baixo peso, Geografia médica, Indicadores sociais 


\section{Introdução}

A ONU e a UNICEF definem baixo peso ao nascer (BPN) como o peso de nascimento abaixo de 2.500 gramas. O peso ao nascer influencia o estado de saúde de uma criança individualmente, em um contexto clínico e também a nível populacio$\mathrm{nal}^{1}$. Crianças nascidas com baixo peso apresentam 20 vezes mais chances de morrer que bebês com peso considerado normal ${ }^{2}$. De acordo com a Declaração Mundial sobre a Sobrevivência, a Proteção e o Desenvolvimento da Criança, a proporção de BPN não deve ultrapassar $10 \%{ }^{3}$.

O baixo peso ao nascer é um fator de risco para a morbidade e mortalidade neonatal e infantil, sua redução pode contribuir para diminuir a mortalidade infantil ${ }^{4}$. Os números da mortalidade materna e neonatal estão relacionados à falta de reconhecimento deste problema como sendo social e político, além do desconhecimento da sua magnitude e da deficiência na qualidade dos serviços de saúde no Brasil ${ }^{5}$. Países de baixa e média renda são os mais atingidos por este problema, concentrando $98 \%$ dos natimortos ${ }^{4}$.

No Brasil, o baixo peso ao nascer apresenta seus maiores percentuais nas regiões de melhor situação socioeconômica, fenômeno denominado paradoxo do baixo peso ao nascer ${ }^{6-8}$. Nas áreas menos desenvolvidas do país, a atenção inadequada e a precariedade de acesso à tecnologia perinatal traz como consequência muitos nascidos vivos pré-termos que morrem logo após o parto que provavelmente são registrados de forma incorreta ou simplesmente não o são, o que pode contribuir para a redução das proporções de prematuridade nestes locais ${ }^{7}$.

Em 2004 um relatório do Ministério da Saúde mostrou que as regiões sul e sudeste conseguiram prevenir o óbito neonatal, pois tinham um maior número de nascidos vivos com baixo peso, indicando assim, uma maior qualidade da assistência à saúde reprodutiva da mulher ${ }^{6}$. Estudo realizado na Região Metropolitana de São Paulo demonstrou a importância da qualidade da assistência ao parto, pois apresentou dados em que partos realizados fora dos hospitais tinham elevadas proporções de neonatos com condições biológicas negativas e mães em situações de vulnerabilidade?.

O baixo peso ao nascer é condicionado ao tempo de gestação e ao crescimento intrauterino, que sofrem influência do estado nutricional da gestante ao iniciar a gravidez e ao longo da mesma, doenças, tabagismo, adequação da assistência pré-natal, idade da gestante e paridade. Tais condições seriam, em parte, consequências da situação socioeconômica que inclui a renda familiar e o nível de escolaridade da gestante ${ }^{10}$.

Estudos realizados em diferentes regiões do Brasil mostram que mães pertencentes a grupos socialmente mais vulneráveis recebem assistência pré-natal de menor qualidade ${ }^{11,12}$. A impossibilidade ou a dificuldade de acesso à assistência pré-natal adequada, por gestantes em situação socioeconômica desfavorecidas, evidencia um grande problema social do Brasil, que culmina em possíveis problemas no decorrer da gestação e no parto ${ }^{13}$.

A discriminação dos mais pobres faz com que estes serviços acentuem as diferenças entre cidadãos ao invés de corrigi-las ${ }^{12}$. Segundo Kilsztajn et al. ${ }^{14}$, para promover a justiça social, é necessário mensurar e atender a demanda por serviços de saúde da população pobre do país .

Organizações internacionais, assim como o governo brasileiro, têm se dedicado a reduzir as desigualdades em saúde e a Mortalidade infantil. Visando diminuir as desigualdades regionais, o Pacto pela Redução da Mortalidade Infantil na Região Nordeste e Amazônia Legal é um exemplo de ação que faz parte de um compromisso maior, proposto pelo governo brasileiro ${ }^{15}$. A distribuição espacial da oferta de serviços de saúde é desigual, sendo os básicos utilizados com maior frequência, gerando menos custo e os complexos requerendo maior tecnologia, maior investimento financeiro e menor demanda ${ }^{16}$. O padrão de consumo dos serviços de saúde é influenciado por vários aspectos da sua oferta, dentre eles o comportamento individual perante o agravo e a localização geográfica dos serviços ${ }^{17}$.

O Brasil vive atualmente em contradição, pois legalmente asseguram-se direitos universais de saúde e ao mesmo tempo são ofertados serviços que não garantem o acesso ou a sua utilização com qualidade e nem de acordo com as necessidades $^{18}$. Regulação pública efetiva e mecanismos financeiros são necessários para que o direito constitucional de universalização do SUS seja garantido, o que guiaria a busca do serviço de acordo com a necessidade da população e não de acordo com o poder de compra ${ }^{17}$.

Diante do exposto, este trabalho pretende conhecer a distribuição espacial das taxas de baixo peso ao nascer em 2009 e sua correlação com os indicadores sociais e de serviços nos Estados brasileiros. 
Estudo do tipo ecológico, cujas unidades de análise de área foram os estados brasileiros, que totalizou 27 elementos na amostra total, considerando os dados de 2009.

Coleta de dados. Utilizaram-se técnicas de análise espacial de área, com dados do Sistema de Informações sobre Nascidos Vivos (SINASC) e Instituto de Pesquisa Econômica e Aplicada (IPEA) para a extração das variáveis independentes e do Instituto Brasileiro de Geografia e Estatística (IBGE) para a coleta das malhas digitais.

Variáveis do Estudo. Foi considerado o baixo peso ao nascer como variável desfecho (número de crianças nascidas com menos de $2.500 \mathrm{~g} /$ total de crianças nascidas vivas) e as seguintes variáveis independentes: fatores relacionados à mãe (proporção de mães maiores de 35 anos de idade: número de mães de nascidos vivos com 35 anos/total de mães de todas as faixas etárias; proporção de mães com menos de 8 anos de estudo: número de mães de nascidos vivos com menos de 8 anos de estudo/total de mães); assistência médico hospitalar (Taxa de consultas de pré-natal: número de mães com 7 consultas ou mais/ número total de mães, Taxa de leitos de UTI neonatal: número de leitos de UTI neonatal/1000 nascidos vivos, Taxa de crianças pré-maturas: número de crianças prematuras/total de nascidos vivos); variável socioeconômica (Renda domiciliar per capita).

Análise dos dados. Foram utilizados os softwares Terra View 4.1.0 ${ }^{19}$ para a construção dos mapas temáticos da distribuição espacial do baixo peso e dos clusters, com os respectivos valores de Moran e o GeoDa 0.9.9.14 ${ }^{20}$ para a avaliação da correlação espacial e construção dos mapas de correlação (análise bivariada).

Foi construído um mapa com as taxas de baixo peso por estado em escala de cinza, com 5 partes iguais, sendo a pior condição apresentada na tonalidade mais escura. Para avaliar a dependência espacial foi utilizado o coeficiente de autocorrelação global de Moran. Quanto à hipótese para o índice de Moran, tem-se que a $\mathrm{H}_{0}$ é de independência espacial e a $\mathrm{H}_{1}$ é de dependência espacial, portanto, para confirmar a hipótese nula, o valor do Moran é zero. Ao verificar a Hipótese da dependência espacial os valores de Moran podem ser positivos (correlação direta; valores semelhantes tendem a se localizar vizinhos) ou negativos (correlação inversa; valores altos estarão cercados de valores baixos, e viceversa).
Utilizou-se também o índice de Moran local (LISA), que determina a dependência dos dados com relação aos seus vizinhos. Este indicador permite identificar padrões de associação espacial que podem caracterizar a ocorrência de agrupamentos (ou clusters) entre os polígonos estudados. Tais dados foram apresentados através dos boxmap (apresenta a presença de clusters sem considerar a significância), Moran map (apresenta os clusters com significância estatística).

A análise bivariada LISA foi usada ainda para verificação da correlação entre a variável desfecho e cada variável independente. A correlação espacial bivariada pode ser considerada complementar (ou mesmo substitutiva) à correlação linear não espacial entre duas variáveis na mesma localidade. Dessa forma, visualiza-se a associação linear entre uma variável $X_{k}$ no local $i, X_{k}{ }_{k}{ }^{i}$ a lag espacial correspondente para a outra variável $\mathrm{Wy}_{1}^{\mathrm{i}}$. Neste caso, uma matriz de pesos padronizada somente pode ser usada uma vez, fornecendo uma interpretação da lag espacial como a "média" dos valores na vizinhança ${ }^{21}$.

A dependência espacial poderá ser direta ou inversa de acordo com o valor do Moran encontrado. E as relações entre os vizinhos nos clusters poderão ser dos tipos: Alto-Alto (altas taxas de BPN e altas taxas da variável independente), Baixo-Baixo (baixas taxas de BPN e baixas taxas da variável independente), Alto-Baixo (altas taxas de BPN e baixas taxas da variável independente), Baixo-Alto (baixas taxas de BPN e altas taxas da variável independente).

\section{Resultados}

A Figura 1 (a) demonstra a distribuição da taxa de baixo peso ao nascer nos estados brasileiros. As maiores taxas se concentraram nos estados da região sul e sudeste. Foi observada ainda a existência de autocorrelação espacial para o baixo peso ao nascer com coeficiente global de Moran de 0,267 e um valor de $\mathrm{p}=0,02$.

Quanto à formação de clusters das taxas de baixo peso ao nascer (Box map), apresentada na Figura 1 (b), foi observado um aglomerado do tipo alto-alto na região Sudeste.

Quanto aos mapas do tipo Box map das variáveis independentes, mostrados na Figura 2, observa-se que a região Sul e Sudeste apresentam resultados diferentes dos encontrados nas regiões Norte e Nordeste. Regiões Sul e Sudeste apresentaram relação alto-alto para as seguintes variáveis: mães com 35 anos ou mais, realização 


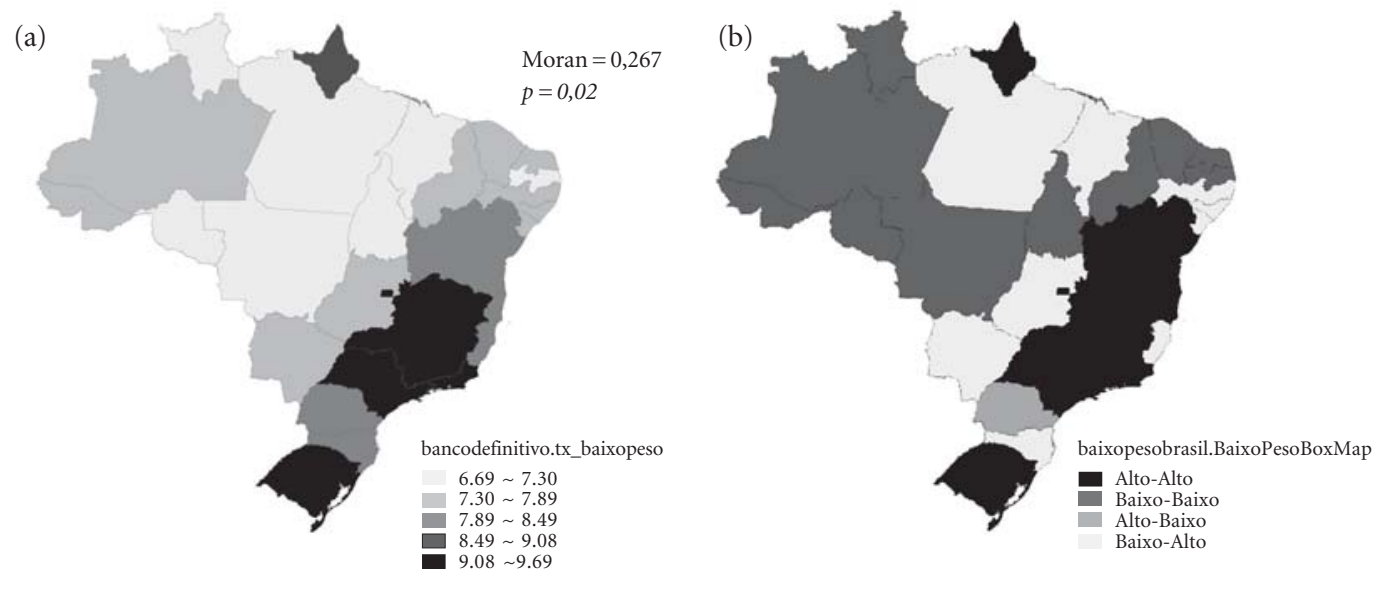

Figura 1. (a) Mapa temático da Taxa de Baixo Peso ao Nascer nos estados brasileiros. (b) Mapa da autocorrelação espacial box map da Taxa de Baixo Peso ao Nascer nos estados brasileiros

(a)

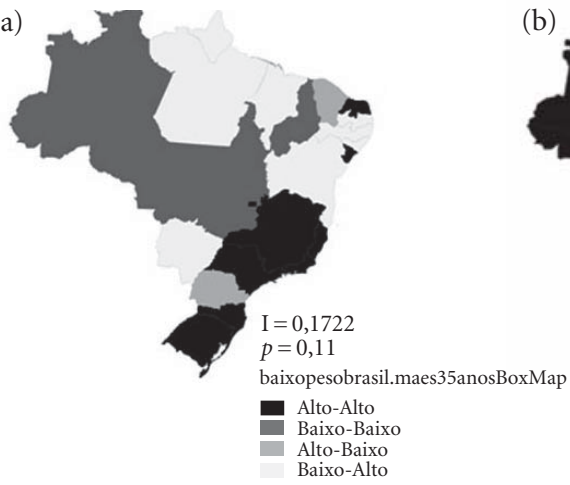

(b)

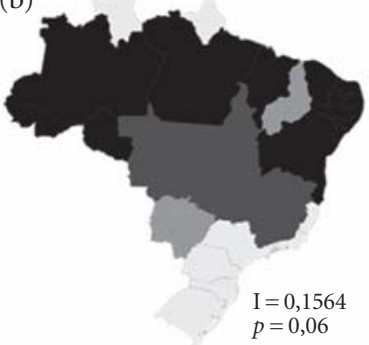

(c)

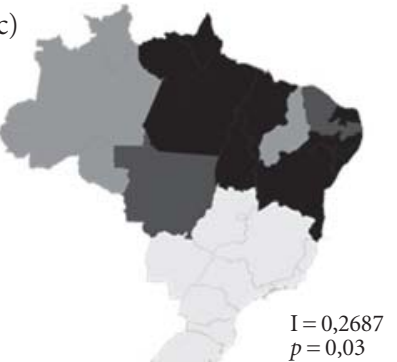

baixopesobrasil.consultasPNBoxMap

baixopesobrasil.maes8aEstudoBoxMap

Alto-Alto
Baixo-Baixo

Alto-Baixo

(d)

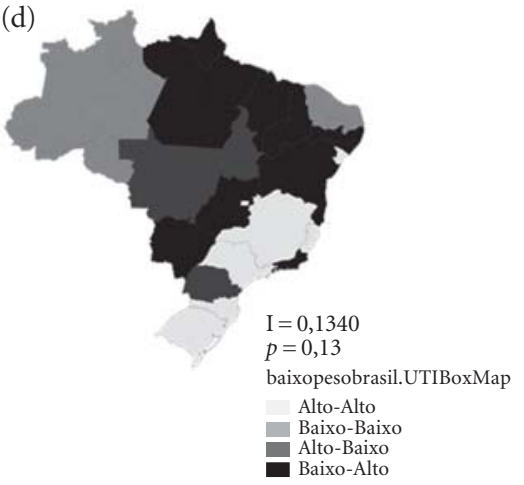

(e)

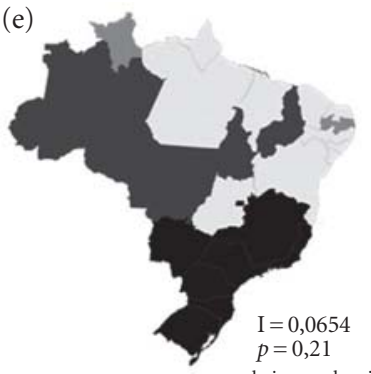

(f)

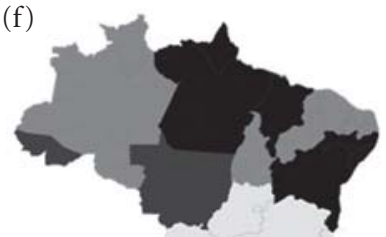

baixopesobrasil.prematuridadeBoxMap

- Alto-Alto

Baixo-Baixo

Alto-Baixo
$\mathrm{I}=0,1578$

$p=0,03$

baixopesobrasil.rendaBoxMap

Alto-Alto

Baixo-Baix

llto-Baixo

Figura 2. (a) Mapa da auto correlação espacial box map da proporção de mães maiores de 35 anos de idade; (b) Mapa da auto correlação espacial box map da proporção de mães com menos de 8 anos de estudo; (c) Mapa da auto correlação espacial box map da taxa de realização de consultas de pré-natal; (d) Mapa da auto correlação espacial box map da taxa de leitos de UTI neonatal; (e) Mapa da auto correlação espacial box map da taxa de crianças prematuras; (f) Mapa da auto correlação espacial box map da renda domiciliar per capita. 


\section{Fatores relacionados à mãe}

Quanto às variáveis que avaliam os fatores relacionados à mãe a Figura 3a apresenta aglomerado com correlação entre baixa taxa de baixo peso ao nascer e baixa taxa de mães maiores de 35 anos ou mais na região Norte.

Quanto a taxa de mães com 8 ou menos anos de estudo (Figura 3b), houve correlação em estados do Nordeste (Baixa taxa de baixo peso ao nascer e alta taxa de mães com 8 ou menos anos de estudo) e entre os estados da região Sudeste (Alta taxa de baixo peso ao nascer e Baixa taxa de mães com 8 ou menos anos de estudo).

\section{Fatores relacionados ao acesso} aos serviços de saúde

A Taxa de realização de 7 consultas ou mais de pré-natal (Figura 4) mostrou correlação em

estados da região Norte, evidenciando baixa taxa de baixo peso ao nascer e baixa taxa de realização de 7 consultas ou mais de pré-natal. Estados da região Sul/Sudeste (Minas Gerais, Rio de Janeiro, São Paulo e Paraná) apresentaram correlação entre as altas taxas de baixo peso ao nascer e altas taxas de realização de 7 consultas ou mais de pré-natal.

\section{Fator socioeconômico}

Na Figura 5 pode-se observar uma correlação entre as baixas taxas de baixo peso ao nascer e baixa renda per capita em estados do norte e nordeste e em um estado do sudeste, alta taxa de baixo peso ao nascer e alta renda domiciliar per capita.

\section{Discussão}

A análise de dados baseados em sistemas de informações geográficas tem sido cada vez mais útil para se compreender a interação entre seres humanos e ambiente; e suas consequências para a saúde ${ }^{22}$. De acordo com os resultados deste trabalho, a distribuição espacial das taxas de baixo peso ao nascer nos estados tem relação com o

Fatores relacionados à mãe
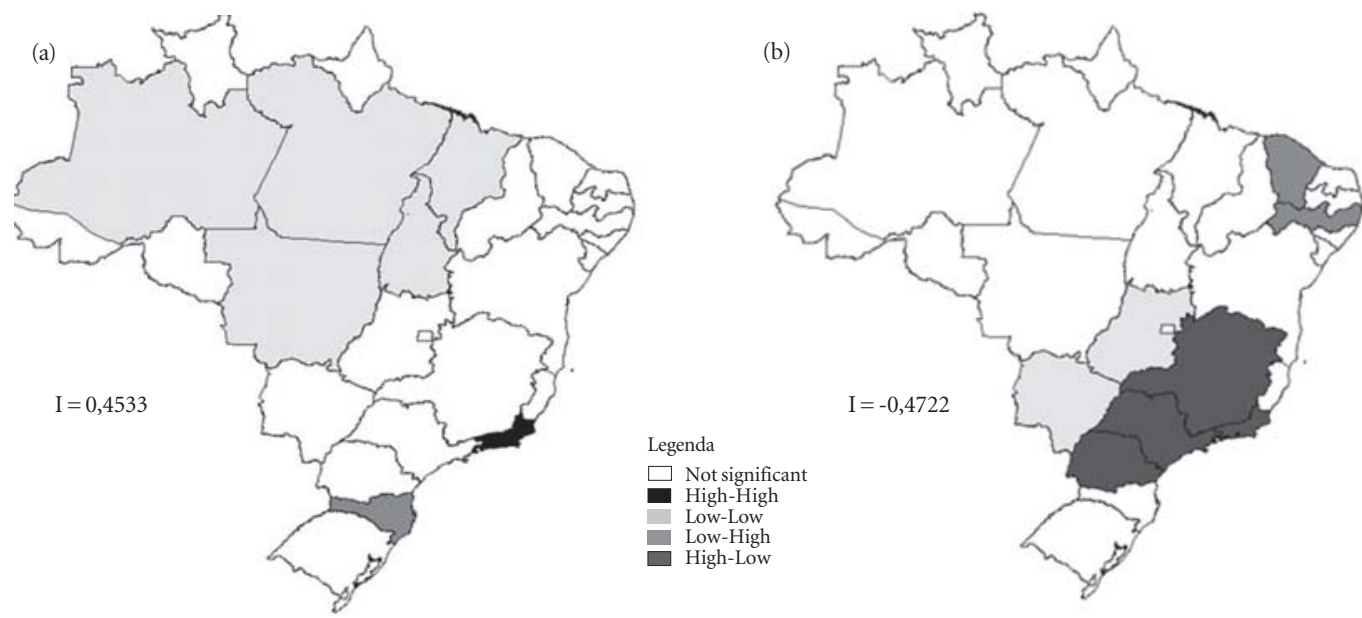

Figura 3. (a) Correlação espacial entre Taxa de Baixo Peso ao Nascer e Taxa de mães com 35 anos ou mais por nascido vivo, Brasil 2009. (b) Correlação entre Taxa de Baixo Peso ao Nascer e Taxa de mães com menos de 8 anos de estudo, Brasil 2009. 
Fatores relacionados ao acesso aos serviços de saúde

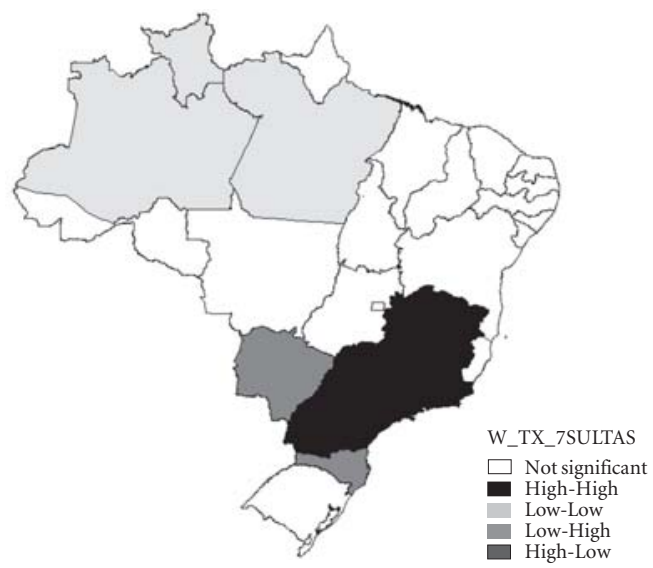

Figura 4. Correlação espacial entre Taxa de Baixo Peso ao Nascer e Taxa de realização de 7 consultas ou mais de pré-natal por nascido vivo, Brasil 2009.

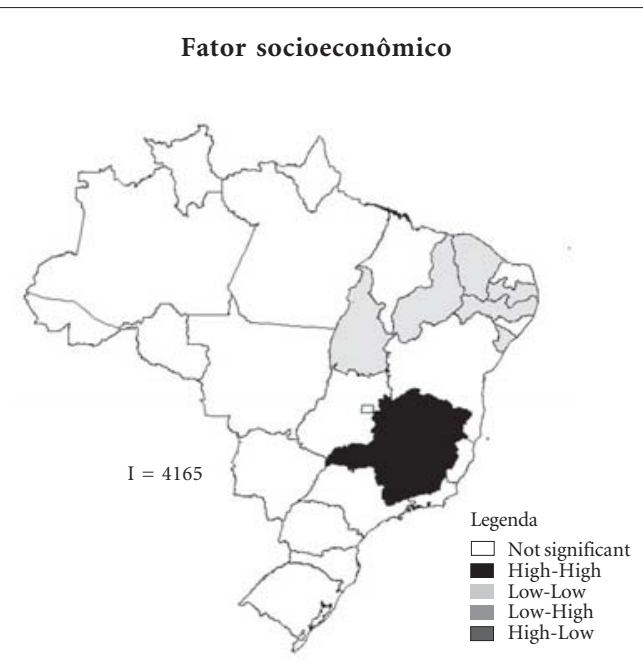

Figura 5. Correlação espacial entre Taxa de Baixo Peso ao Nascer e Renda per capita, Brasil 2009.

espaço e evidencia a desigualdade social e em saúde vivenciada no território brasileiro. Os estados da região Sul e Sudeste apresentaram os melhores indicadores socioeconômicos e as maiores taxas de BPM.

Em 2008 e 2010 autores com objetivo de analisar as desigualdades da proporção do baixo peso ao nascer no Brasil também mostraram resultado semelhante, e a esse fenômeno denominaram de paradoxo do baixo peso ao nascer ${ }^{7,8}$. Da mesma forma, de 1996 a 1999, os estados de Minas Gerais, Rio de Janeiro, São Paulo e Rio Grande do Sul apresentaram as maiores taxas de baixo peso ao nascer dentre todos os estados brasileiros ${ }^{23}$ Tais resultados foram interpretados com cautela, tendo em vista que esses estados fazem parte das regiões mais desenvolvidas do país e com melhores níveis de saúde. Devido à fragilidade do sistema naquele momento, os autores atribuíram esses achados a uma deficiência da cobertura do Sistema de Informação sobre Nascidos Vivos (SINASC) nas regiões de pior nível de saúde.

No entanto, a cobertura desse sistema hoje é adequada e a qualidade da informação melhorou bastante nos últimos anos ${ }^{24}$, sendo considerada uma relevante fonte de dados para a pesquisa e a avaliação em saúde ${ }^{25}$. A utilização dos dados disponíveis nesse sistema tem sido essencial para a vigilância e o monitoramento das desigualdades em saúde e a qualidade da assistência ${ }^{26}$.

Quando comparado ao Sistema de Informação de Mortalidade (SIM), o SINASC foi melhor avaliado, demonstrando ao longo dos anos um aumento na sua cobertura, principalmente nas regiões Sudeste, Sul e Centro-Oeste ${ }^{27,28}$. O indicador: "Razão entre nascidos vivos informados e estimados", que mede a relação quantitativa entre nascidos vivos informados no SINASC e estimados por projeções demográficas, é utilizado nesse tipo de avaliação. Certamente que é necessário aperfeiçoar cada vez mais a cobertura e a qualidade das informações sobre nascidos vivos, principalmente em regiões menos desenvolvidas o que possibilitaria a análise das informações com maior veracidade ${ }^{7}$.

A despeito da discussão acerca das deficiências do SINASC, as diferenças regionais observadas nas taxas de baixo peso ao nascer parecem ter maior relação com os serviços de saúde perinatais e intervenções médicas do que com as condições sociais ${ }^{8}$. A melhoria da qualidade do diagnóstico e do atendimento às gestantes vem possibilitando a indução do parto e os partos do tipo cesarianas ${ }^{29}$. Há assim, aumento de nascimentos pré-termo e das proporções de baixo peso ao nascer, diminuindo dessa forma os números da mortalidade infantil, principalmente nas regiões que oferecem melhores serviços de saúde perinatais.

Por outro lado, a atenção inadequada e a precariedade do acesso à tecnologia perinatal em áreas menos favorecidas resulta em mortes de 
nascidos vivos logo após o parto, com consequente subregistro ou registro com classificação equivocada, diminuindo, portanto, as proporções de prematuridade e de nascimentos com baixo peso ${ }^{7,29}$.

Os resultados encontrados, no que diz respeito à assistência médico hospitalar (Taxa de crianças pré-maturas, Taxa de consultas de prénatal, Taxa de leitos de UTI neonatal), corroboram com as afirmações acima, na medida em que comprovam que em locais onde a oferta e o acesso aos serviços são restritos, como nas regiões norte e nordeste, há um menor número de recém-nascidos de baixo peso. Ao mesmo tempo, essas são as regiões com situação socioeconômica mais precária e maiores índices de mortalidade infantil do país.

Quanto à prematuridade, foi observada distribuição espacial semelhante com as altas taxas de BPN nos estados da região sul e sudeste, o que está de acordo com os resultados de Moraes et al. ${ }^{30}$ e os de Monteiro et al. ${ }^{31}$, que constataram essa relação mesmo em se tratando de famílias com melhores condições socioeconômicas.

A realização de consultas de pré-natal foi menor nos estados do Norte e está em consonância com outros estudos, nos quais foi constatada que a baixa cobertura está concentrada nas regiões com baixos indicadores socioeconômicos e níveis inferiores de desenvolvimento ${ }^{32}$, assim como a inadequação do uso da assistência pré-natal também foi maior para as mulheres atendidas em hospitais públicos e com menores níveis de escolaridade e de renda familiar ${ }^{11}$.

Igualmente, a quantidade de leitos de UTI neonatal foi menor nos estados das regiões Norte e Nordeste. Ceará, Pernambuco e Acre apresentaram forte correlação entre baixas taxas de baixo peso ao nascer e baixo número de leitos de UTI neonatal por mil nascidos vivos. Nesse sentido, as taxas de baixo peso ao nascer são mascaradas pelas de mortalidade neonatal, maiores nessas regiões ${ }^{33}$.

A necessidade de leitos intensivos neonatais é determinada pelo número de nascidos vivos, a qualidade da assistência pré-natal, a frequência de nascimentos prematuros, o baixo peso e o tempo de permanência sob cuidados intensivos neonatais ${ }^{33}$. Apesar desses critérios epidemiológicos, o que se observa no Brasil é uma forte concentração dos serviços de maior complexidade nas regiões mais desenvolvidas do país.

Segundo Magalhães et al. ${ }^{34}$, a implementação de políticas públicas se constitui processo complexo e dinâmico que utiliza estratégias que visam amenizar conflitos, além de lançar compromissos. Para se fazer cumprir os princípios do SUS, deve haver oferta serviços de saúde de forma universal, equânime e integral, no entanto, não é o que se observa ao estudar a oferta e a distribuição de serviços de saúde nas regiões brasileiras. $\mathrm{Na}$ região Norte há falhas na oferta de serviços de saúde com precariedade das redes intermunicipais de assistência, inexistem equipamentos e instalações físicas e os recursos humanos são insuficientes para o atendimento básico ${ }^{16}$.

É do Estado a maior responsabilidade na busca do desenvolvimento social e econômico de todas as unidades federativas, no entanto o que se observa historicamente é uma grande desigualdade regional. A qual se dá principalmente devido ao momento atual da economia mundial, onde as localidades com maior infraestrutura, mão de obra qualificada e melhor qualidade de vida da população deslocam as atividades econômicas, enquanto que as áreas excluídas desse mercado tendem a permanecer à margem dos fluxos econômicos, apresentando menores níveis de renda e bem estar ${ }^{35}$. Diante dessa tendência, as políticas públicas têm o papel de fomentar o desenvolvimento nacional mais equanime atuando na busca do equilíbrio, disseminando o desenvolvimento nacional.

Diante desses achados pode-se inferir que as dinâmicas socioespaciais não têm sido pautadas no momento da formulação das políticas setoriais. As políticas de saúde são frágeis no que tange às questões regionais e seria importante fortalecer a dimensão territorial no âmbito do SUS visando enfrentar nacionalmente as desigualdades ${ }^{36}$.

No que se refere às variáveis socioeconômicas avaliadas no estudo, a renda domiciliar per capita e a escolaridade materna apresentaram correlação espacial inversa com o baixo peso ao nascer, evidenciando menores taxas de baixo peso em estados com menor renda domiciliar per capita (região nordeste) e as maiores em estados com altas taxas de escolaridade materna (região sul e sudeste). Como as taxas de mortalidade infantil nos estados do nordeste ainda são elevadas, a baixa concentração de baixo peso ao nascer é um fator de alerta, pois provavelmente recém-nascidos com baixo peso não estão conseguindo sobreviver.

Apesar da redução das taxas de mortalidade infantil ao longo dos anos, as regiões Norte e Nordeste ainda se mantêm com as taxas mais elevadas do país, principalmente a neonatal. $\mathrm{Na}$ região Norte, o deslocamento geográfico para o parto, o nível socioeconômico, porte populacio- 
nal e a localização do município de residência apresentaram relação com os níveis de mortalidade infantil ${ }^{37}$.

Vale salientar que a variável renda per capita per si não representa necessariamente piores condições de saúde, o mais importante é conhecer a forma como essa renda está distribuída. Nesse sentido, a concentração de renda e a pobreza são o maior desafio das políticas públicas. Atualmente, a busca pela erradicação da pobreza e da marginalização através da redução das desigualdades sociais e regionais, perpassa pela Política de Transferência Condicionada de Renda, introduzida no Brasil nos anos $1990^{38}$. O Programa Bolsa Família é o principal programa de transferência condicionada de renda do Brasil e tenta aproximar os beneficiários de um conjunto de políticas sociais (educação, saúde e assistência social) ${ }^{39}$.

A desigualdade de renda tem um impacto direto sobre o bem-estar social; está diretamente relacionada ao nível de diversas variáveis socioeconômicas importantes como, por exemplo, a taxa de poupança da economia, a taxa de mortalidade infantil e a extensão da pobreza ${ }^{40}$. Uma situação econômica desfavorável acarreta saúde mais precária e, consequentemente, uma pior saúde relaciona-se a menor rendimento. Indivíduos mais pobres utilizam menos o serviço de saúde, apesar de terem uma maior necessidade, acentuando as desigualdades financeiras. Políticas de saúde podem, neste sentido, combater a pobreza e a desigualdade ${ }^{41}$.

Outro indicador avaliado foi a idade materna. Estados da região da Amazônia Legal formaram um aglomerado com baixas taxas de mães com 35 anos ou mais e baixas taxas de baixo peso ao nascer. Essa questão deve ser avaliada tanto do ponto de vista biológico como social. Estudo demonstra que os extremos da idade reprodutiva, aliados à baixa escolaridade, estão associados ao baixo peso ao nascer ${ }^{42}$. Do ponto de vista social, é sabido que há uma maior prevalência de mães adolescentes em regiões com baixas condições socioeconômi$\mathrm{cas}^{32}$, o que justifica uma menor taxa de mães com idade maior que 35 anos na região norte.

O Brasil, país de dimensões continentais e com situações díspares em suas regiões, deve tentar solucionar seus problemas utilizando-se de políticas com foco na integração nacional. Espaços em que o desenvolvimento não consiga ser atingido devido à dinâmica capitalista devem ser incluídos nas políticas públicas, visando à redu- ção das desigualdades através do desenvolvimento de atividades econômicas, inserção da população no mercado de trabalho, implantação de infraestrutura e de serviços básicos de saúde, educação e outros ${ }^{35}$. Políticas públicas efetivas, com ênfase na educação, saúde, saneamento, habitação e de distribuição de renda são essenciais para a redução da desigualdade no Brasil, e é fundamental que o poder público realize tais ações.

Apesar das diferenças regionais ainda muito evidentes, particularmente nas taxas de baixo peso ao nascer encontradas nesse estudo, há atualmente uma tendência geral positiva dos indicadores sociais e de desigualdade, em especial os que tratam da educação e da saúde ${ }^{43}$. Acompanhadas das políticas de distribuição de renda se faz necessário ainda um forte investimento na melhoria da rede de assistência médica que é essencial para a redução da mortalidade neonatal, evento comum em situações de risco como o baixo peso ao nascer.

Outra questão essencial para a reflexão dos resultados encontrados diz respeito ao tipo do desenho de estudo. Investigações com dados agregados requerem indicadores válidos e confiáveis, particularmente quando se trata da aferição das desigualdades sociais em saúde ${ }^{34}$. Em função da complexidade da estrutura social da sociedade brasileira, os indicadores utilizados podem ser insuficientes na busca da compreensão das relações sociais existentes, não se conseguindo muitas vezes contemplar a sua verdadeira dimensão ${ }^{44}$.

Da mesma forma, nos estudos ecológicos com a utilização dos Sistemas de Informações Geográficas (SIG), a seleção da escala geográfica, ou seja, a definição espacial das fronteiras das áreas deve ser feita com cautela. Isso porque ao se avaliar uma mesma área considerando diferentes fronteiras - por exemplo, setor censitário, município ou estado - pode-se obter resultados diferentes, efeito este conhecido como "problema da unidade de área modificável”. O presente trabalho utilizou o polígono estado como unidade de análise e isso pode ter encoberto diferenças intraestaduais.

Além dessas limitações, nos estudos agregados existe o fenômeno denominado nas ciências sociais de "falácia ecológica" que é relacionado aos efeitos de escala e de agregação de áreas que podem gerar coeficientes de correlação inteiramente diferentes no indivíduo ${ }^{45}$. 


\section{Conclusões}

A distribuição espacial das taxas de baixo peso ao nascer apresentou-se de forma desigual no território brasileiro. A desigualdade espacial do baixo peso ao nascer reflete as condições socioeconômicas dos estados brasileiros, que foram marcados historicamente por políticas públicas que desrespeitam as dinâmicas sociais e espaciais, além da dinâmica capitalista que deixa à margem dos fluxos econômicos as áreas que não ofereçam infraestrutura e mão de obra qualificada, refletindo na saúde e na vida das pessoas. Depois de verificar que as regiões mais desenvolvidas do país apresentam maiores taxas de baixo peso ao nascer, concluímos então que a presença do serviço e sua utilização pela população fazem diminuir os números da mortalidade infantil e aumentar as taxas de baixo peso ao nascer.

\section{Colaboradores}

MCBM Lima escreveu o artigo; GS Oliveira, CO Lyra e AG Roncalli trabalharam na concepção e revisão do artigo. MAF Ferreira participou na revisão crítica do artigo e aprovação final da versão a ser publicada.

\section{Referências}

1. Noronha GA, Lima MC, Lira PIC, Veras AACA, Gonçalves FCLSP, Malaquias BF. Evolução da assistência materno-infantil e do peso ao nascer no Estado de Pernambuco em 1997 e 2006. Cien Saude Colet 2012; 17(10):2749-2756.

2. World Health Organization (WHO), United Nations Children's Fund (Unicef). Low birthweiight: contry, regional and global estimates. New York: WHO, Unicef; 2004.

3. Organização das Nações Unidas (ONU). Declaração Mundial sobre a Sobrevivência, a Proteção e o Desenvolvimento da Criança. Nova Iorque: ONU; 1990.

4. Lawn JE, Cousens S, Zupan J. 4 million neonatal deaths: when? Where? Why? Lancet 2005; 365(9462): 891-900.

5. Brasil. Ministério da Saúde (MS). Pacto pela Redução da Mortalidade Infantil Nordeste-Amazônia Legal: diagnóstico no Brasil. [site na Internet]. [acessado 2012 jun 10]. Disponível em: http://portal. saude.gov.br/portal/saude/profissional/area.cfm?Id _area $=1583$

6. Brasil. Ministério da Saúde (MS). Saúde Brasil 2004: uma análise da situação de saúde. Cap 2. Saúde reprodutiva: gravidez, assistência pré-natal, parto e baixo peso ao nascer. [site na Internet]. [acessado 2012 mar 15]. Disponível em: http://portal. saude.gov.br/portal/arquivos/pdf/capitulo2_sb.pdf

7. Andrade CLT, Szwarcwald CL, Castilho EA. Baixo peso ao nascer no Brasil de acordo com as informações sobre nascidos vivos do Ministério da Saúde, 2005. Cad Saude Publica 2008; 24(11):2564-2572.
8. Silva AAM, Silva LM, Barbieri MA, Bettiol H, Carvalho LM, Ribeiro VS, Goldani MZ. The epidemiologic paradox of low birth weight in Brazil. Rev Saude Publica 2010; 44(5):767-775.

9. Silva ZP, Almeida MF, Ortiz LP, Alencar GP, Alencar AP, Schoeps D, Minuci EG, Novaes HMD. Características dos nascidos vivos, das mães e mortalidade neonatal precoce na Região Metropolitana de São Paulo, Brasil. Cad Saude Publica 2009; 25(9):1981-1989.

10. Monteiro CA, Benicio MHD, Ortiz LP. Tendência secular do peso ao nascer na cidade de São Paulo (19761998). Rev Saude Publica 2000; 34(Supl. 6):26-40.

11. Coimbra LC, Silva AAM, Mochel EG, Alves MTSSB, Ribeiro VS, Aragão VMF, Bettiol H. Fatores associados à inadequação do uso da assistência prénatal. Rev Saude Publica 2003; 37(4):456-462.

12. Gonçalves CV, Cesar JA, Mendoza-Sassi RA. Qualidade e eqüidade na assistência à gestante: um estudo de base populacional no Sul do Brasil. Cad Saude Publica 2009; 25(11):2507-2516.

13. Minagaw AAT, Biagoline REM, Fujimori E, Oliveira IMV, Moreira APCA, Ortega LDS. Baixo peso ao nascer e condições maternas no pré-natal. Rev. esc. enferm. 2006; 40(4):548-554.

14. Kilsztajn S, Camara MB, Carmo MSN. Gasto privado com saúde por classes de renda. Rev. Assoc. Med. Bras 2002; 48(3):258-262.

15. Garcia LP, Santana LR. Evolução das desigualdades socioeconômicas na mortalidade infantil no Brasil, 1993-2008. Cien Saude Colet 2011; 16(9):3717-3728. 
16. Rodrigues CG, Simões RF, Amaral PV. Distribuição da rede de oferta de serviços de saúde na região norte: uma análise espacial multivariada. [texto para discussão] 2007. [acessado 2012 abr. 12]. Disponíve em: http://www.cedeplar.ufmg.br/pesquisas/td/TD\% 20308.pdf

17. Travassos C, Viacava F, Fernandes C, Almeida CM. Desigualdades geográficas e sociais na utilização de serviços de saúde no Brasil. Cien Saude Colet 2000; 5(1):133-149.

18. Fleury S. Desigualdades injustas: o contradireito à saúde. Psicol. Soc. 2011; 23(Esp.):45-52.

19. Terraview [computerprogram]. Version 4.1.0. Instituto Nacional de Pesquisas Espaciais (INPE); 2011.

20. OpenGeoDa [computer program]. Version 0.9.9.14 Geoda Center for geospatial analysis and computation and Arizona Board of Regents; 2011.

21. Anselin L, Syabri I, Smirnov O. Visualizing Multivariate Spatial Correlation with Dynamically Linked Windows. 2002 [documento da Internet] [acessado 2013 fev 3]. Disponível em: http://citeseerx. ist.psu.edu/viewdoc/summary?doi=10.1.1.118.7163

22. Ricketts CT. Geographic information systems and public health. Annu. Rev. Public. Health. 2003; 24:1-

23. Mello Jorge MHP, Gotlieb SLD, Laurenti R. Situação de saúde no Brasil: análise do período 1996 a 1999. Brasília: OPS, OMS; 2002.

24. Garcia LP, Santana LR. Evolução das desigualdades socioeconômicas na mortalidade infantil no Brasil, 1993-2008. Cien Saude Colet 2011; 16(9):37173728.

25. Paiva NS, Coeli CM, Moreno AB, Guimarães RM, Camargo JKR. Sistema de informações sobre nascidos vivos: um estudo de revisão. Cien Saude Colet 2012; 16(1):1211-1220.

26. Predebon KM, Mathias TAF, Aidar T, Rodrigues AL. Desigualdade sócio-espacial expressa por indicadores do Sistema de Informações sobre Nascidos Vivos (SINASC). Cad Saude Publica 2010 26(8) 1583-1594

27. Organização Panamericana de Saúde (OPAS). Indicadores básicos para a saúde no Brasil: conceitos e aplicações. Brasília: OPAS; 2008.

28. Andrade CLT, Szwarcwald, CL. Desigualdades sócio-espaciais da adequação das informações de nascimentos e óbitos do Ministério da Saúde, Brasil, 2000-2002. Cad Saude Publica 2007; 23(5):1207-1216.

29. Silva LR, Christoffel MM, Souza KV. História, conquistas e perspectivas no cuidado à mulher e à criança. Texto contexto enferm 2005; 14(4):585-593.

30. Moraes AB, Zanini RR, Giugliani ERJ, Riboldi J. Tendência da proporção de baixo peso ao nascer, no período de 1994-2004, por microrregião do Rio Grande do Sul, Brasil: uma análise multinível. Cad Saude Publica 2011; 27(2):229-240.

31. Monteiro CA, Benicio MHD, Ortiz LP. Tendência secular do peso ao nascer na cidade de São Paulo (1976-1998). Rev Saude Publica, 2000; 34(6):26-40 .

32. Melo EC, Mathias TAF. Spatial Distribution and Self-Correlation of Mother and Child Health Indicators in the State of Parana, Brazil. Rev. LatinoAm. Enfermagem 2010; 18(6):1177-1186.
33. Sociedade Brasileira De Pediatria (SBP). Departamento Científico de Neonatologia. Estimativa da necessidade de leitos de UTI neonatal no Brasil. [acessado 2012 maio 8]. Disponível em: http://www. sbp.com.br

34. Magalhães R, Burlandy L, Senna M, Schottz V, Scalercio G. A implementação do programa Bolsa Família: as experiências de São Francisco de Itabapoana e Duque de Caxias. Cien Saude Colet 2007; 12(6):1513-1524

35. Brasil. Ministério da Integração Nacional. Política Nacional De Desenvolvimento Regional. Brasília: Ministério da Integração Nacional; 2005.

36. Viana ALD, Machado CV, Baptista TWF, Lima LD, Mendonça MHM, Heimann LS, Albuquerque MV Iozzi FL, David VC, Ibañez P, Frederico S. Sistema de saúde universal e território: desafios de uma política regional para a Amazônia Legal. Cad Saude Publica 2007; 23(Supl. 2):S117-S131.

37. Almeida WS, Szwarcwald CL. Mortalidade infantil e acesso geográfico ao parto nos municípios brasileiros. Rev Saude Publica 2012; 46(1):68-76.

38. Monteiro DAA; Ferreira MAM; Teixeira KMD Determinantes da gestão do Programa Bolsa Família: análise do índice de gestão descentralizada em Minas Gerais. Saude soc 2009; 18(2):214-226.

39. Magalhaes R, Bodstein R. Avaliação de iniciativas e programas intersetoriais em saúde: desafios e aprendizados. Cien Saude Colet 2009; 14(3):861-868.

40. Barros RP, Mendonca RSP. Os determinantes da desigualdade no Brasil. Rio de Janeiro: Instituto de Pesquisa Econômica Aplicada; 1995. (Textos para discussão) [documento da Internet]. [acessado 10 out 2012]. Disponível em: http://www.ipea.gov.br/ pub/td/1995/td_0377.pdf.

41. Neri M, Soares W. Desigualdade social e saúde no Brasil. Cad Saude Publica 2002; 18(1):S77-S87.

42. Coutinho PR, Cecatti JG, Surita FG, Souza JP, Morais SS. Factors associated with low birth weight in a historical series of deliveries in Campinas, Brazil. Rev. Assoc. Med. Bras 2009; 55(6):692-699.

43. Luna FV, Klein HS. Desigualdade e indicadores sociais no Brasil. In: Schwartzman LF, Schwartzman IF, Schwartzman FF, Schwartzman ML, organizadores. O Sociólogo e as Políticas públicas: Ensaios em Homenagem a Simon Schwartzman. Rio de Janeiro: Editora FGV; 2009. Cap. 5. p. 97-116.

44. Ferreira MAF, Latorre MRDO. Desigualdade social e os estudos epidemiológicos: uma reflexão. Cien Saude Colet 2012; 17(9):2523-2531.

45. Câmara G, Carvalho MS, Cruz OG, Correa V. Análise espacial de áreas. INPE, 2002 [documento da Internet]. [acessado 2012 nov 10]. Disponível em: http://mtc-m12.sid.inpe.br/col/sid.inpe.br/sergio/ 2005/02.17.14.42/doc/cap5-areas1\%5b1\%5d.pdf.

Artigo apresentado em 23/11/2012

Aprovado em 05/02/2013

Versão final apresentada em 24/02/2013 